\title{
Tapinarof Cream 1\% Once Daily for the Treatment of Moderate to Severe Atopic Dermatitis in Children and Adults: The Pivotal Phase 3 ADORING Clinical Program
}

Lawrence F. Eichenfield, ${ }^{1,2}$ Jonathan I. Silverberg, ${ }^{3}$ Robert Bissonnette, ${ }^{4}$ Anna M. Tallman, ${ }^{5}$ Philip M. Brown, ${ }^{5}$ David S. Rubenstein, ${ }^{5}$ Stephen C. Piscitelli, ${ }^{5}$ John E. Jett ${ }^{5}$

\section{SYNOPSIS}

Atopic dermatitis $(A D)$ is a chronic, relapsing, and remitting inflammatory skin disease characterized by intense pruritus and eczematous lesions that can substantially impact sleep and quality of life ${ }^{1-4}$

In the US, approximately 16.5 million adults and 9.6 million children under the age of 18 years have $A D^{5}$

There is a need for efficacious non-steroidal topical therapies for $A D$ without restrictions on duration, extent or site of use

Tapinarof is a novel, first-in-class, small-molecule topical therapeutic aryl hydrocarbon receptor modulating agent (TAMA) in development for the treatment of $A D$ and psoriasis. Tapinarof has demonstrated efficacy and a psemittive effect in Phase 3 clinical trials for the treatment of plaque psoriasis: PSOARING 1 (NCT03956355), PSOARING 2 (NCT03983980), and PSOARING 3 (NCT04053387)

Tapinarof specifically binds to and activates the aryl hydrocarbon receptor (AhR), a ligand-dependent transcription factor. This leads to the downregulation of inflammatory Th2 cytokines (including interleukin [IL]-4, IL-5 and (L-13), increase in expression of skin barrier proteins reated to keratinocyte differentiation, including filaggrin,

loricrin, and involucrin, and antioxidant activity ${ }^{6-10}$ (Figure 1)

Figure 1. Potential Mechanisms of Action of Tapinarof in Atopic Dermatitis

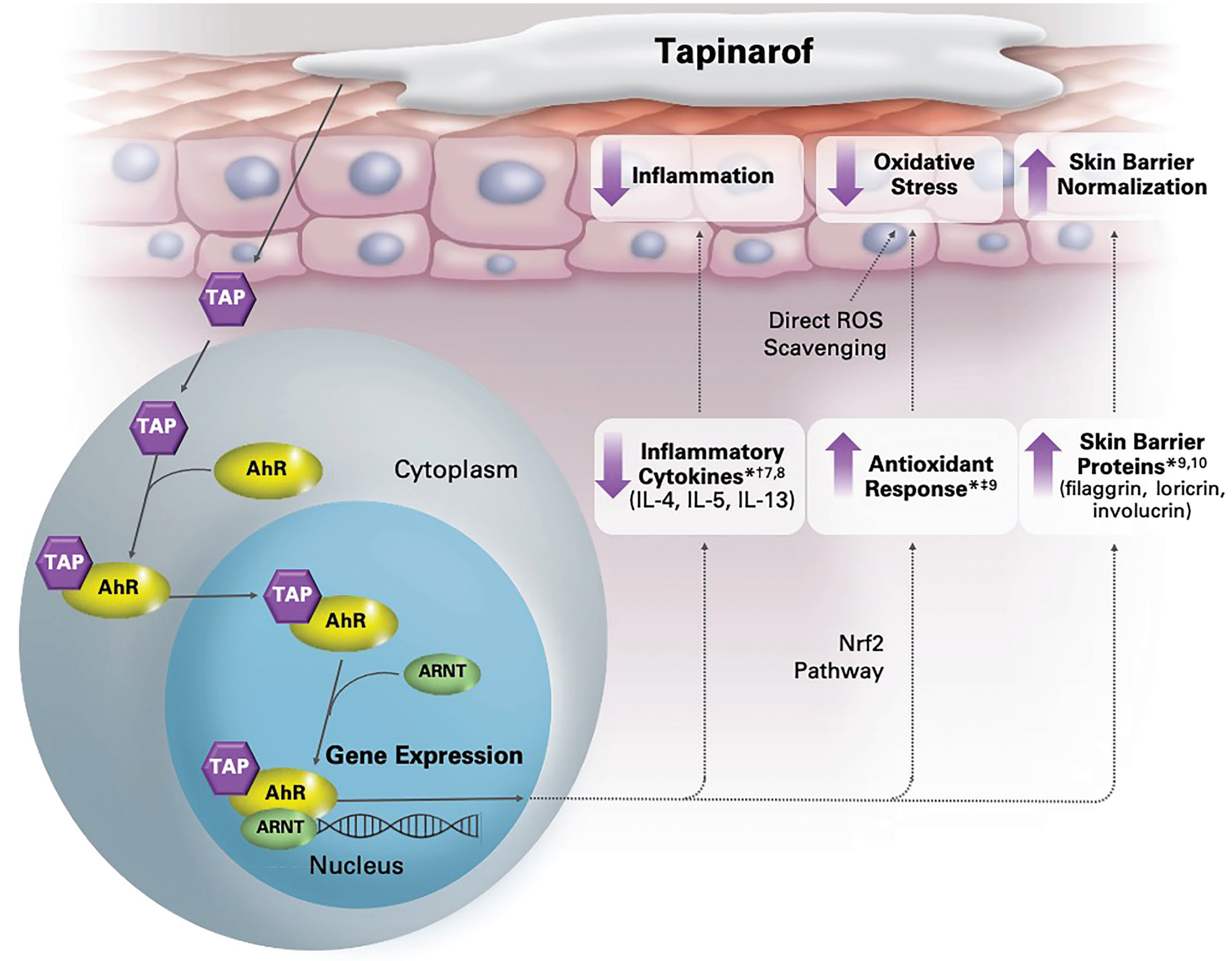

"Demonstrated in vitro. Demonstated in mouse models. "Demonstrated ex vivo. AhR, aryl hydrocarbon receptor; ARNT, ary hydrocarbon receptor nuclear translocator; IL, interleukin; Nrf2, nuclear factor erythroid 2-related factor 2;

Tapinarof cream $1 \%$ once daily (OD) demonstrated significant efficacy versus vehicle at 12 weeks and was well tolerated in adolescents and adults with moderate to severe AD in a Phase 2b trial (NCT02564055). Efficacy was generally maintained through the last study visit, 4 weeks after completing treatment, warranting further investigation a potential remittive effect: this will be defined as the maintenance of disease control (a validated Investigato Global Assessment for Atopic Dermatitis ${ }^{\mathrm{TM}}[\mathrm{VI}$ GA-ADTM score of 0 [clear] or 1 [almost clear]) off therapy

\section{OBJECTIVE}

To assess the efficacy and safety of tapinarof cream 1\% OD in children and adults with moderate to severe $A D$ in the wo pivotal Phase 3 studies (ADORING 1 and 2) and a longterm extension Phase 3 trial (ADORING 3)

\section{METHODS}

Trial Design: ADORING 1 and 2

ADORING 1 and ADORING 2 are two identically designed, Phase 3, multicenter (US and Canada),

double-blind, vehicle-controlled randomized trials (Figure 2)

Following a 30-day screening period, patients aged $\geq 2$ years old with an vIGA-AD score $\geq 3$ (moderate to severe) and a percentage body surface area (\%BSA) affected of $\geq 5-\leq 35 \%$ will be randomized 2:1 to tapinarof cream $1 \%$ OD or vehicle QD for 8 weeks

Figure 2. Trial Design: ADORING 1 and ADORING 2

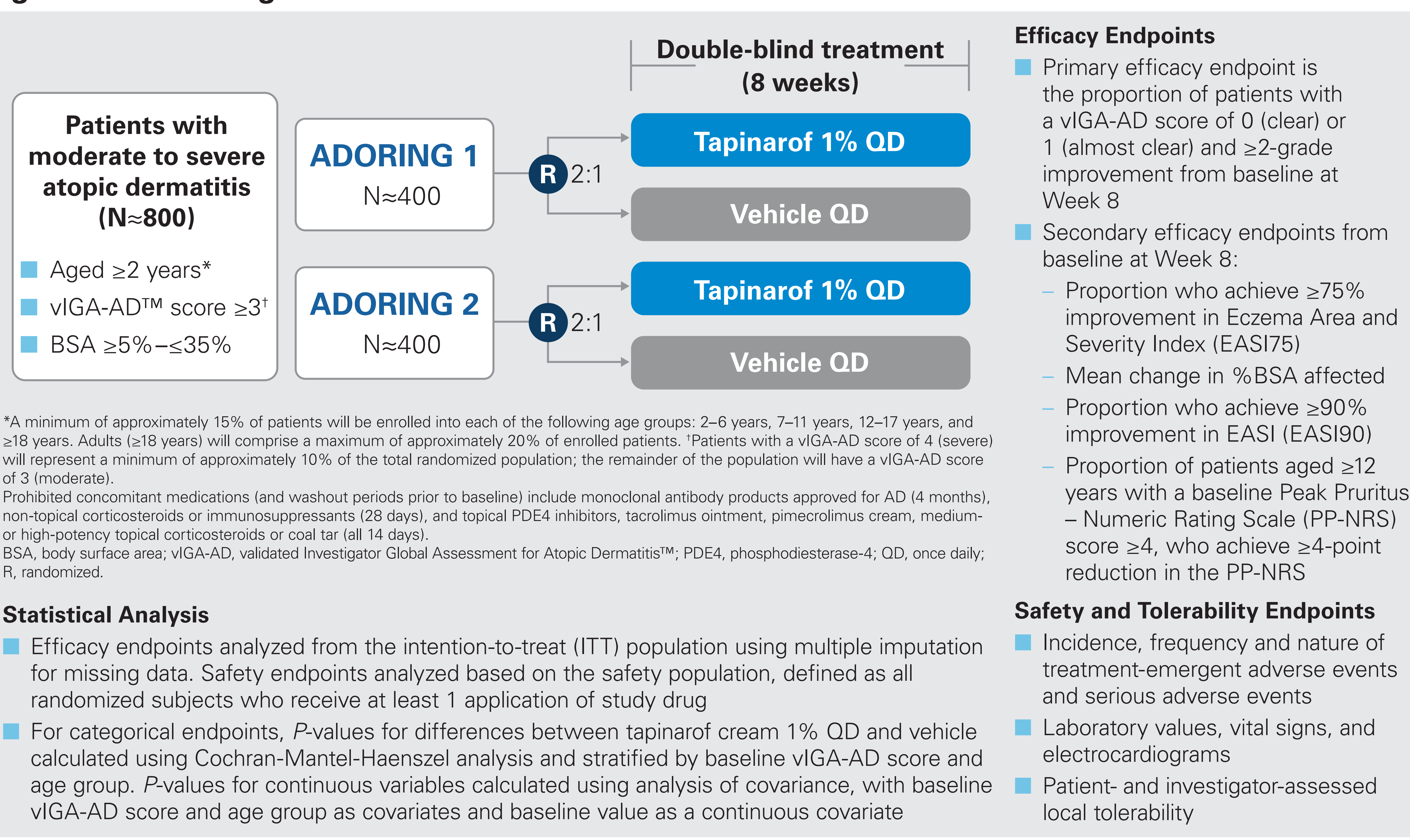

Trial Design: ADORING 3

ADORING 3 is a long-term, open-label, multicenter extension trial to evaluate the long-term safety and efficacy of tapinarof $1 \%$ QD in patients with AD (Figure 3 )

Eligible patients completing ADORING 1, ADORING 2, or the Maximal Use Pharmacokinetics trial can enroll in ADORING 3

In addition, approximately 125 pediatric patients (aged 2 to $<18$ years) can enroll directly in ADORING 3 if they had a vIGA-AD score of $\geq 3$ (moderate) and \%BSA affected $\geq 40 \%$ at screening and baseline (pre-randomization), or patients with a vIGA-AD score of 2 (mild) at screening and baseline (prerandomization) regardless of \%BSA affected, and were thus not eligible for participation in the ADORING 1 and 2 pivotal trials

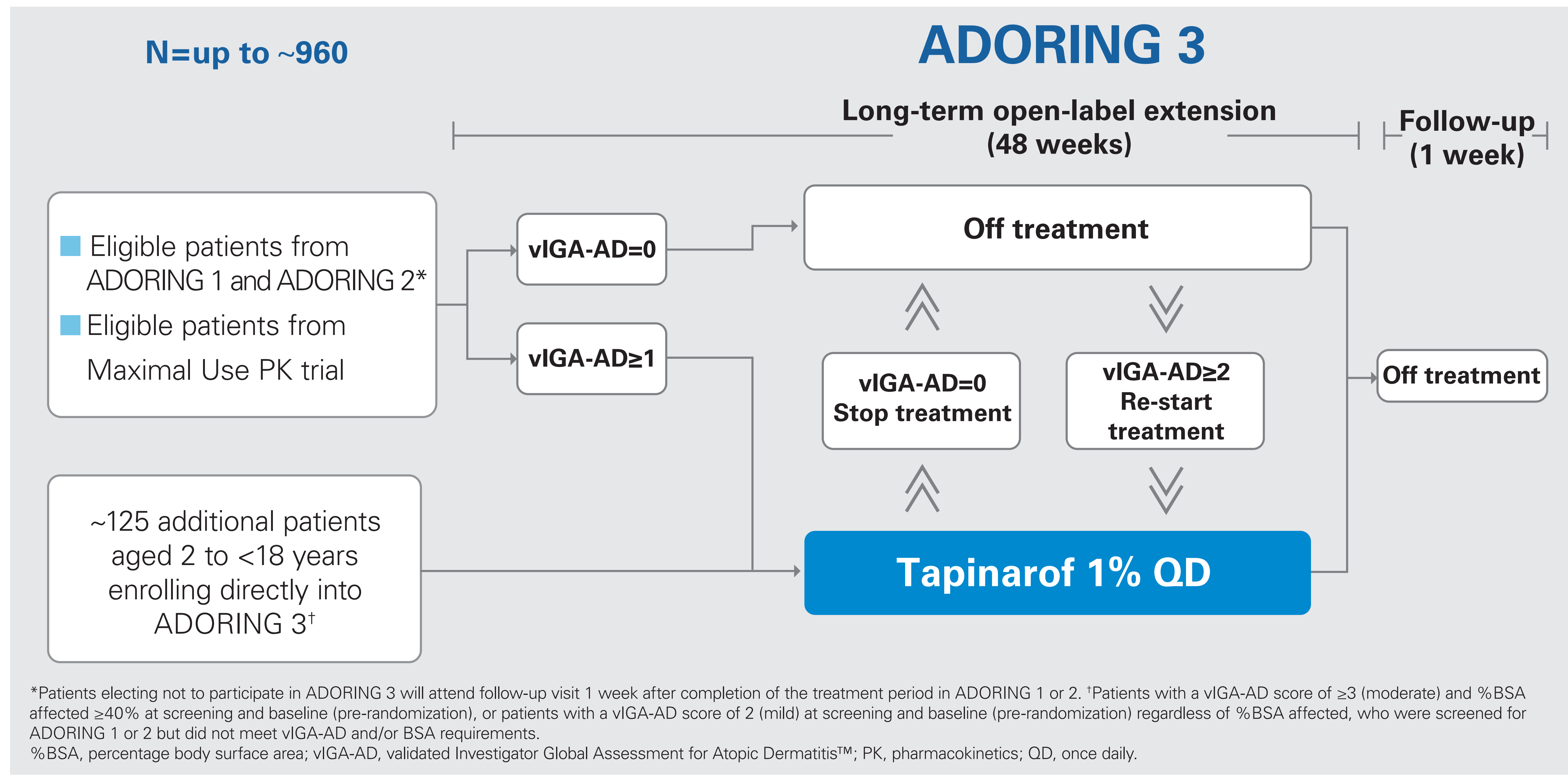

Figure 3. Trial Design: ADORING 3

In ADORING 3, patients will be treated based on their vIGA-AD score:

Patients entering with, or achieving, a vIGA-AD score of 0 (clear) will discontinue treatment and will be monitored for remittive effect, defined as off therapy maintenance of a vIGA-AD score of 0 (clear) or 1 (almost clear)

Patients entering with a vIGA-AD score $\geq 1$ (almost clear) will receive tapinarof 1\% QD until they achieve complete disease clearance, defined as a vIGA-AD score of 0 (clear)

\section{METHODS (continued)}

Trial Design: ADORING 3 (continued)

If disease worsening occurs (defined as a vIGA-AD score

$\geq 2$ [mild]), tapinarof $1 \%$ QD will be started and continued until a vIGA-AD score of 0 (clear) is achieved

Treatment and re-treatment will continue until the end of the study

Endpoints and Statistical Analysis: ADORING 3

Safety and tolerability endpoints: Adverse events, patient- and investigator-assessed local tolerability, laboratory values, vital signs, and physical exams

Efficacy endpoints include:

Complete disease clearance: Proportion of patients achieving vIGA-AD of 0 (clear)

Remittive effect: Duration of efficacy maintenance, (clear) or 1 (almost clear) while off therapy, after achieving complete disease clearance $(\mathrm{vIGA}-\mathrm{AD}=0)$ Response: Proportion of patients who enter the trial with a vIGA-AD $\geq 2$ (mild) and achieve a vIGA-AD of 0 (clear) or almost clear (1)

Durability of response (absence of tachyphylaxis on therapy): Maintenance of efficacy on treatment

Efficacy endpoints will be based on the ITT population using observed case and last observation carried forward Vafety endpoint analysis will be based on the ITT population

\section{CONCLUSIONS}

This comprehensive Phase 3 clinical trial program will assess the efficacy, safety, tolerability, durability, and potential remittive effect of tapinarof cream $1 \%$ QD for the treatment of moderate to severe $A D$ in patients down to 2 years of age

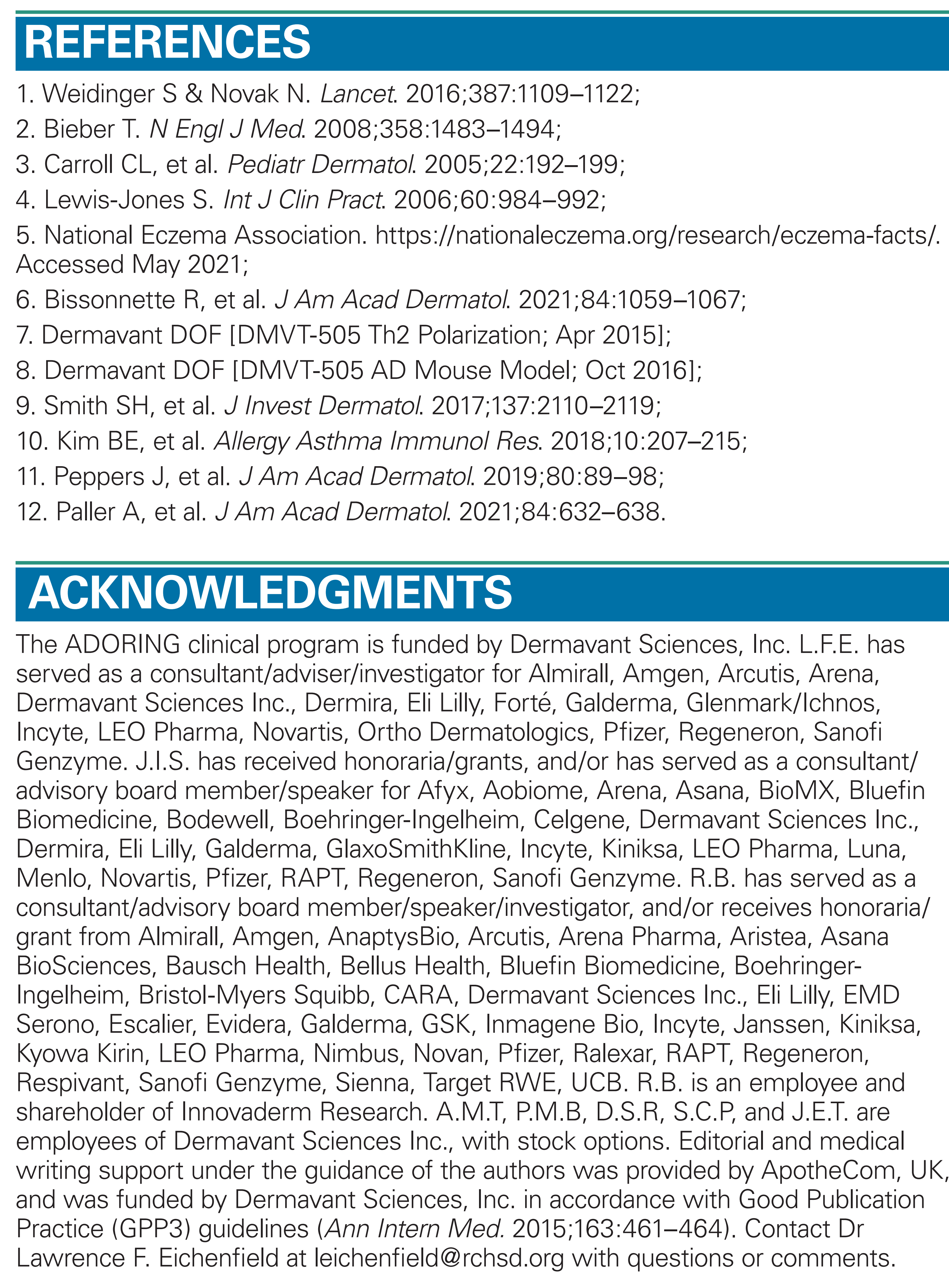

